\title{
Infrared and Submillimetre Observing Conditions on the Antarctic Plateau
}

\author{
Marton G. Hidas, Michael G. Burton, Matthew A. Chamberlain \\ and John W. V. Storey \\ School of Physics, University of New South Wales, NSW 2052, Australia \\ mgh@roen.phys.unsw.edu.au
}

Received 2000 January 23, accepted 2000 May 19

\begin{abstract}
The Antarctic Plateau provides the best terrestrial sites for infrared (IR) and submillimetre (sub-mm) astronomy. In this paper we examine the relative importance of temperature, aerosol content and precipitable water vapour to determine which parameters have the greatest influence on atmospheric transmission and sky brightness. We use the atmospheric modelling program MODTRAN to model the observed sky spectrum at the South Pole from the near-IR to the sub-mm. We find that temperature and aerosol content determine the quality of near-IR observing conditions, aerosol content is the determining factor in the mid-IR up to $20 \mu \mathrm{m}$, while at longer wavelengths, including the sub-mm, it is the water vapour content that matters. Finding a location where aerosol levels are minimised is a key constraint in determining the optimum site on the Antarctic Plateau for an IR observatory.
\end{abstract}

Keywords: site testing—atmospheric effects—infrared: general

\section{Introduction}

It is now well established that the Antarctic Plateau can provide the best terrestrial astronomical observing conditions across the IR and sub-mm wavebands (e.g. Nguyen et al. 1996; Ashley et al. 1996; Chamberlin, Lane \& Stark 1997; Phillips et al. 1999; Chamberlain et al. 2000; Ade et al. 2000). It is well understood that this is a result of the extremely dry and cold air, and the high elevation of the Plateau (over $3000 \mathrm{~m}$ ). As an example, at the South Pole in winter, temperatures fall below $-70^{\circ} \mathrm{C}$ and the precipitable water vapour in the atmosphere is below $250 \mu \mathrm{m}$. This leads to extremely dark skies, as summarised in Burton 1998. At $2.4 \mu \mathrm{m}$ the sky brightness can fall below $100 \mu \mathrm{Jy} \operatorname{arcsec}^{-2}(20-100$ times less than at temperate sites), at $3.6 \mu \mathrm{m}$ to $\sim 100$ $\mathrm{mJy} \operatorname{arcsec}^{-2}(\sim 20$ times less than at temperate sites), at $11 \mu \mathrm{m}$ to $\sim 20 \mathrm{Jy} \mathrm{arcsec}^{-2}$ ( 10 times lower), and at $350 \mu \mathrm{m}$ to $\sim 60 \mathrm{Jy} \operatorname{arcsec}^{-2}$ (a reduction of two). At the highest points of the Plateau, such as Domes A and C, conditions are expected to be even more favourable, due to further reductions in temperature and water vapour content. These sites also offer improved seeing, due to lower wind speeds and a thinner inversion layer. The improvements in the IR and sub-mm observing conditions compared to those at temperate sites are surmised to arise from three principal effects: low temperature, low water vapour content and low levels of particulates. The low temperature results in a reduction of the background sky emission. The low water vapour improves the atmospheric transmission and correspondingly decreases its emissivity. The reduced particulate content of the atmosphere also reduces its emissivity compared to that at a mid-latitude site. However, these effects, while they are interrelated, vary significantly with wavelength. In this paper we apply an atmospheric modelling program to compare their contributions and thus quantify their effects.

\section{MODTRAN Modelling}

The atmospheric modelling program MODTRAN 4.0 (AFRL/VSBM) was used to determine the flux from the sky at zenith as a function of wavelength. MODTRAN calculates the transmission and emission of the atmosphere at wavelengths from the optical to the sub-mm. It uses various standard atmospheric models, based on common geographic locations. It also allows the user to define an atmospheric profile with any specified set of parameters. The MODTRAN package also includes several parameters for the definition of aerosol profiles in the atmosphere. The most important of these are the type of aerosol and the visibility. Several types of aerosol are available, based on common aerosol mixtures that are found in most terrestrial conditions, e.g. rural, urban and maritime. Of all the aerosol types available in MODTRAN, 'radiative fog' was found to produce a mid-IR emission spectrum that was most similar to that measured (Chamberlain et al. 2000). The second main aerosol parameter, visibility, is used to define the amount of the aerosol in the atmosphere.

Data from meteorological balloon launches from the South Pole were used to define the pressure, temperature and relative humidity profiles of our models (see CMDL/NOAA). The profiles for other atmospheric constituents $\left(\mathrm{O}_{3}, \mathrm{CH}_{4}, \mathrm{~N}_{2} \mathrm{O}, \mathrm{CO}, \mathrm{CO}_{2}, \mathrm{O}_{2}, \mathrm{NO}, \mathrm{SO}_{2}\right.$, $\mathrm{NO}_{2}, \mathrm{NH}_{3}, \mathrm{HNO}_{3}$ ) were set to those from MODTRAN's standard 'subarctic winter' model, which was closest to the conditions at the South Pole. However, these have a negligible effect on the sky emission in the IR windows discussed here. (MODTRAN allows the contributions of individual species to the total emission to be examined.) Figure 1 shows the pressure, temperature and relative humidity recorded on 1998 August 11, a typical example of a clear day. The strong temperature inversion in the lowest hundred metres is clearly defined, the temperature rising by $30^{\circ} \mathrm{C}$. Note that while the relative 

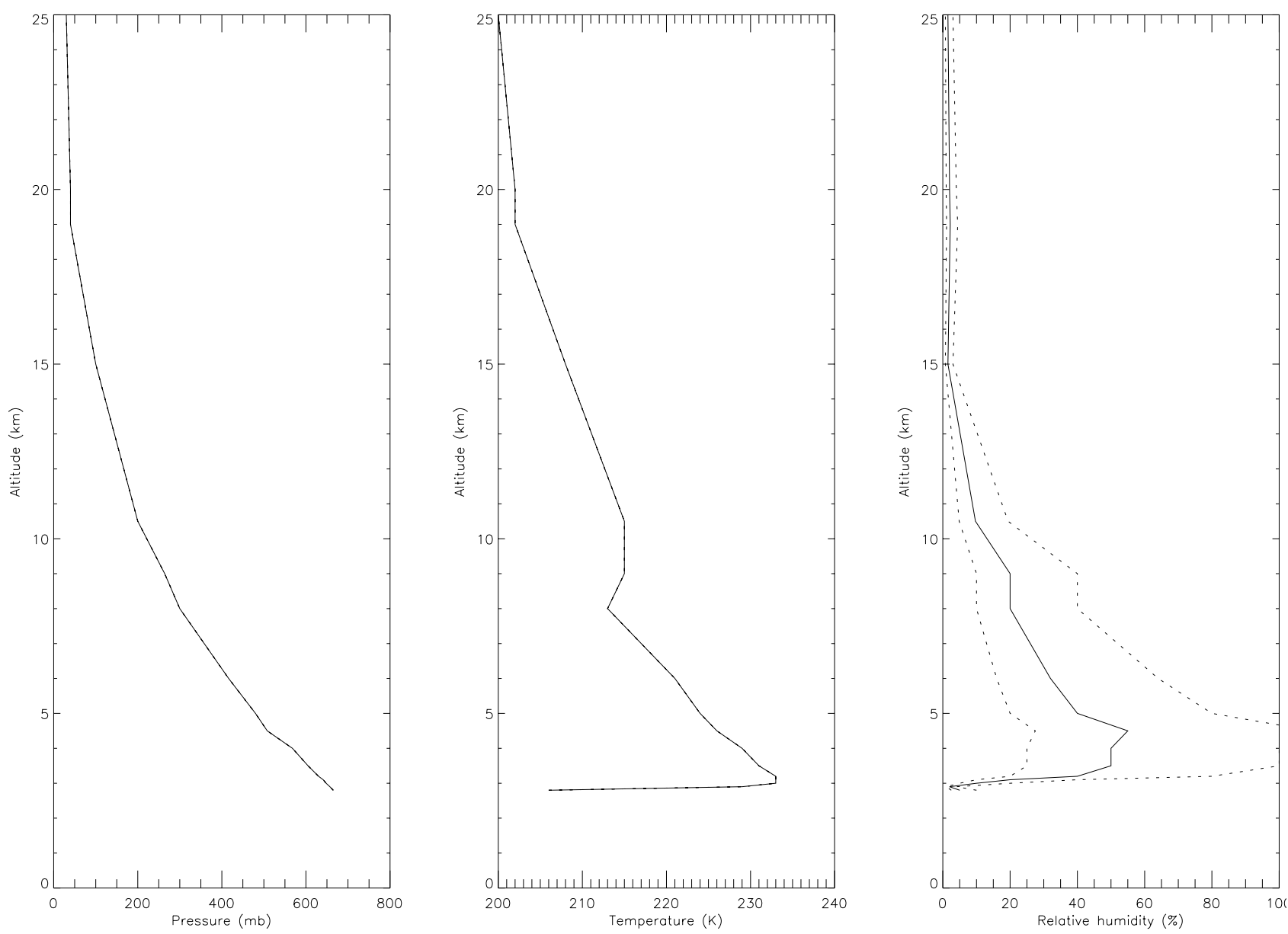

Figure 1-Atmospheric profiles showing the pressure, temperature and relative humidity as functions of altitude above the South Pole. These profiles were used to investigate the fluxes across the IR and sub-mm windows at the site. The model is from Chamberlain et al. (2000), based on radiosonde balloon measurements. The two dotted lines represent doubling and halving the relative humidity. Ice-level is at $2900 \mathrm{~m}$.

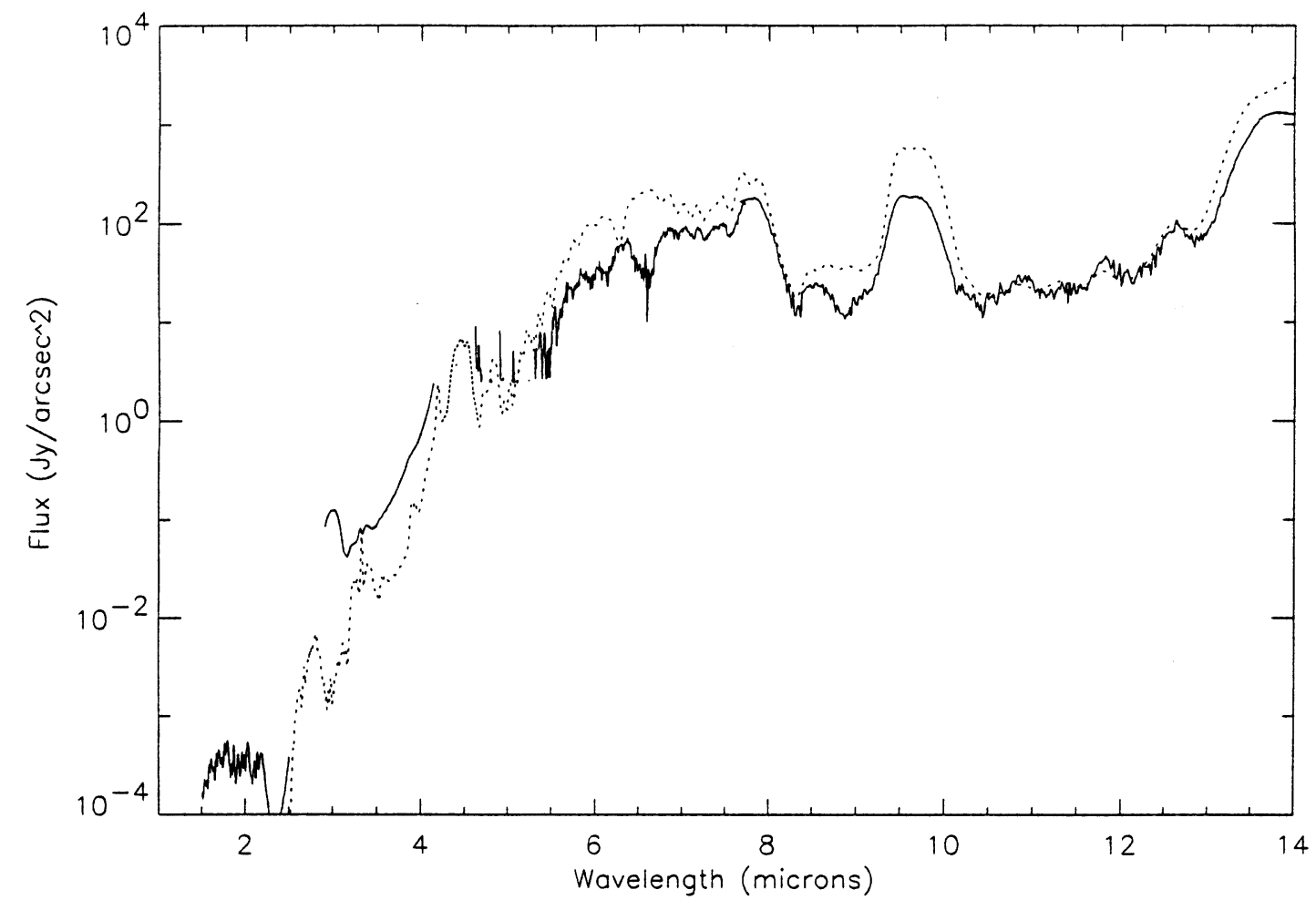

Figure 2-The measured near-IR sky spectrum (from 1.5-2.5 $\mu \mathrm{m}$ and 2.9-4.1 $\mu \mathrm{m}$, Phillips et al. 1999) and mid-IR sky spectrum (5$14 \mu \mathrm{m}$, Chamberlain et al. 2000) at the South Pole. Overplotted as a dashed curve is the model spectrum calculated by MODTRAN, using the measured atmospheric profile of Figure 1 (corresponding to $164 \mu \mathrm{m}$ of precipitable $\mathrm{H}_{2} \mathrm{O}$ ), plus an aerosol visibility of $100 \mathrm{~km}$. The model fails below $2.3 \mu \mathrm{m}$ because of the neglect of airglow emission. 
Table 1. Effect of temperature change on IR and sub-mm fluxes

\begin{tabular}{lcccc}
\hline Temperature & \multicolumn{3}{c}{ Flux $\left(\mathrm{Jy} \operatorname{arcsec}^{-2}\right)$} \\
& $2.4 \mu \mathrm{m}$ & $3.8 \mu \mathrm{m}$ & $11 \mu \mathrm{m}$ & $350 \mu \mathrm{m}$ \\
\hline $10^{\circ} \mathrm{C}$ & $4.3 \times 10^{-2}$ & $2.6 \times 10^{1}$ & $7.0 \times 10^{3}$ & $1.4 \times 10^{2}$ \\
$0^{\circ} \mathrm{C}$ & $2.0 \times 10^{-2}$ & $1.6 \times 10^{1}$ & $5.9 \times 10^{3}$ & $1.3 \times 10^{2}$ \\
$-40^{\circ} \mathrm{C}$ & $4.6 \times 10^{-4}$ & $1.5 \times 10^{0}$ & $2.6 \times 10^{3}$ & $1.1 \times 10^{2}$ \\
$-7^{\circ} \mathrm{C}$ & $1.0 \times 10^{-5}$ & $1.4 \times 10^{-1}$ & $1.1 \times 10^{3}$ & $1.0 \times 10^{2}$ \\
Typical measured flux at South Pole & $1.0 \times 10^{-4}$ & $1.0 \times 10^{-1}$ & $2.0 \times 10^{1}$ & $6.0 \times 10^{1}$ \\
Inferred emissivity & $20 \%$ & $7 \%$ & $1 \%$ & $50 \%$ \\
\hline
\end{tabular}

humidity may seem high, the absolute level of water content is low because of the extreme cold. The total column of precipitable water vapour for this day is $164 \mu \mathrm{m}$. These profiles were assumed to be representative of good observing conditions. Our aim was to determine the effects of water content and aerosol content on the sky emissivity during such times. Also shown in Figure 1 are the profiles obtained when the relative humidity was both doubled and halved, corresponding to total columns of $324 \mu \mathrm{m}$ and $82 \mu \mathrm{m}$ respectively (the column has not doubled for the first case since the humidity actually saturates at $100 \%$ for part of the column). As described later, these two models were used to examine how water vapour content affects the brightness of sky emission.

In Figure 2 are shown measured near-IR (1995) and mid-IR (1998) sky fluxes at the South Pole (from Phillips et al. 1999 and Chamberlain et al. 2000), overlaid with a model spectrum from MODTRAN, calculated for the atmospheric profile of Figure 1. In addition, a 'radiative fog' aerosol layer with visibility equal to $100 \mathrm{~km}$ was included. While not a perfect fit, the model provides a good explanation (see Chamberlain et al. 2000) for the overall flux level and features in the spectrum across the mid-IR. The success of this model was used as a basis for further investigation of the effects of water vapour and aerosols on the complete spectrum from 2.4 to $500 \mu \mathrm{m}$. It fails below $2.3 \mu \mathrm{m}$ because airglow emission is not included.

\section{The Effect of Temperature}

The temperature of the atmosphere affects the flux levels in the near-IR, mid-IR and sub-mm quite differently. Table 1 gives a quantitative indication of these differences at four representative wavelengths by listing the value of the Planck function at four different temperatures. These are chosen as typical values for the atmosphere above mid-latitude sites such as Siding Spring $\left(10^{\circ} \mathrm{C}\right)$ and Mauna Kea $\left(0^{\circ} \mathrm{C}\right)$ and above the South Pole $\left(-40^{\circ} \mathrm{C}\right.$ at the top of the inversion layer and $-70^{\circ} \mathrm{C}$ at the surface). Also listed are measured fluxes at the South Pole and the inferred emissivities for the atmosphere.

At these temperatures, the near-IR flux is on the Wien side of the Planck function, and hence shows the strongest dependence on temperature. Reductions of two orders of magnitude should be obtainable at the Pole at $2.4 \mu \mathrm{m}$. However, as Ashley et al. (1996) and Nguyen et al. (1996) report, the observed reduction is usually only by a factor of $\sim 20$. The cause of this discrepancy appears to be airglow emission (Phillips et al. 1999). In fact, at wavelengths below $2.3 \mu \mathrm{m}$, airglow actually dominates over thermal emission from the atmosphere. On the other hand, at $3.8 \mu \mathrm{m}$ the measured background levels are actually $\sim 20$ times less than at temperate sites, wheareas a simple estimate from the blackbody fluxes would suggest a drop of just a factor of 10 . In the midIR the difference is even more marked, the measured reduction being a factor of 10 compared to an expectation of just 2-3 times based on the temperature drop alone. Clearly the drop in temperature relative to that at a mid-latitude site is not the only factor causing the reduction in background fluxes in the near- and midIR at the South Pole. In the sub-mm the temperature drop has only a small influence on the level of the background, and is not responsible for the 50\% drop in flux at the Pole compared to that at a mid-latitude site.

Values of the Planck function in Jy $\operatorname{arcsec}^{-2}$ are listed for a range of temperatures and wavelengths, representative of temperate and Antarctic observing sites. Shown for comparison are measurements of the sky flux from the South Pole and the inferred emissivity for the atmosphere. The sensitivity of the near-IR emission to temperature implies that even at an ideal site, small variations in the temperature of the atmosphere will cause large changes in the background flux. Near-IR astronomy therefore requires a site that is not only cold, but also has as stable a temperature as possible. In contrast, sub-mm, and even mid-IR, observations are not so strongly affected by local variations in temperature.

\section{The Effect of Water Content}

The starting point for the following analysis is the model shown in Figure 1, obtained from radiosonde data taken on a clear day. The total precipitable water vapour for this model is $164 \mu \mathrm{m}$, which is close to the lowest value measured at the South Pole during winter (Van Allen, Murcray \& Liu 1996). This model did not include any aerosols. The spectrum was calculated for the original 
Table 2. Model sky fluxes (in Jy arcsec ${ }^{-\iota}$ ) averaged over various spectral regions, showing the effects of varying precipitable water vapour content and aerosol visibility

\begin{tabular}{|c|c|c|c|c|c|c|}
\hline \multirow[t]{2}{*}{$\begin{array}{l}\text { Wavelength } \\
\text { range }(\mu \mathrm{m})\end{array}$} & \multicolumn{6}{|c|}{$\begin{array}{l}\text { Precipitable water vapour / Aerosol visibility } \\
\qquad(\mu \mathrm{m}) /(\mathrm{km})\end{array}$} \\
\hline & $164 / \infty$ & $82 / \infty$ & $324 / \infty$ & $164 / 100$ & $164 / 10$ & $164 / 1$ \\
\hline $3.0-3.1$ & $2.2 \times 10^{-3}$ & $1.3 \times 10^{-3}$ & $3.6 \times 10^{-3}$ & $3.1 \times 10^{-3}$ & $1.4 \times 10^{-2}$ & $3.3 \times 10^{-2}$ \\
\hline $3.6-3.8$ & $7.0 \times 10^{-3}$ & $6.5 \times 10^{-3}$ & $8.0 \times 10^{-3}$ & $2.9 \times 10^{-2}$ & $3.0 \times 10^{-1}$ & $6.5 \times 10^{-1}$ \\
\hline $3.0-4.0$ & $2.1 \times 10^{-2}$ & $2.0 \times 10^{-2}$ & $2.2 \times 10^{-2}$ & $3.3 \times 10^{-2}$ & $2.0 \times 10^{-1}$ & $4.2 \times 10^{-1}$ \\
\hline $4.9-5.1$ & $1.2 \times 10^{0}$ & $8.3 \times 10^{-1}$ & $1.8 \times 10^{0}$ & $1.7 \times 10^{0}$ & $8.1 \times 10^{0}$ & $2.2 \times 10^{1}$ \\
\hline $8.2-9.2$ & $2.5 \times 10^{1}$ & $2.4 \times 10^{1}$ & $2.7 \times 10^{1}$ & $3.3 \times 10^{1}$ & $1.3 \times 10^{2}$ & $6.2 \times 10^{2}$ \\
\hline $10.2-11.2$ & $1.3 \times 10^{1}$ & $1.3 \times 10^{1}$ & $1.3 \times 10^{1}$ & $2.2 \times 10^{1}$ & $1.6 \times 10^{2}$ & $9.5 \times 10^{2}$ \\
\hline $10.0-13.0$ & $2.5 \times 10^{1}$ & $2.4 \times 10^{1}$ & $2.7 \times 10^{1}$ & $4.3 \times 10^{1}$ & $2.5 \times 10^{2}$ & $1.4 \times 10^{3}$ \\
\hline $18.0-22.0$ & $5.5 \times 10^{2}$ & $3.8 \times 10^{2}$ & $8.0 \times 10^{2}$ & $6.0 \times 10^{2}$ & $1.2 \times 10^{3}$ & $3.8 \times 10^{3}$ \\
\hline $20.0-20.2$ & $8.1 \times 10^{1}$ & $4.1 \times 10^{1}$ & $1.6 \times 10^{2}$ & $1.3 \times 10^{2}$ & $7.7 \times 10^{2}$ & $3.7 \times 10^{3}$ \\
\hline $24.1-24.9$ & $4.3 \times 10^{2}$ & $2.2 \times 10^{2}$ & $8.0 \times 10^{2}$ & $4.6 \times 10^{2}$ & $9.1 \times 10^{2}$ & $3.3 \times 10^{3}$ \\
\hline $32.0-32.5$ & $2.4 \times 10^{3}$ & $1.5 \times 10^{3}$ & $3.5 \times 10^{3}$ & $2.4 \times 10^{3}$ & $2.6 \times 10^{3}$ & $3.4 \times 10^{3}$ \\
\hline $220-230$ & $2.0 \times 10^{2}$ & $1.5 \times 10^{2}$ & $2.4 \times 10^{2}$ & $2.0 \times 10^{2}$ & $2.0 \times 10^{2}$ & $2.0 \times 10^{2}$ \\
\hline $330-370$ & $4.9 \times 10^{1}$ & $3.8 \times 10^{1}$ & $6.6 \times 10^{1}$ & $4.9 \times 10^{1}$ & $4.9 \times 10^{1}$ & $5.1 \times 10^{1}$ \\
\hline $430-470$ & $2.7 \times 10^{1}$ & $2.0 \times 10^{1}$ & $3.8 \times 10^{1}$ & $2.7 \times 10^{1}$ & $2.7 \times 10^{1}$ & $2.8 \times 10^{1}$ \\
\hline
\end{tabular}

Table 3. Model atmospheric transmission, averaged over various spectral regions, showing the effects of varying precipitable water vapour content and aerosol visibility

\begin{tabular}{ccccccc}
\hline $\begin{array}{c}\text { Wavelength } \\
\text { range }(\mu \mathrm{m})\end{array}$ & \multicolumn{7}{c}{$\begin{array}{c}\text { Precipitable water vapour / Aerosol visibility } \\
(\mu \mathrm{m}) /(\mathrm{km})\end{array}$} \\
\cline { 2 - 7 } & $164 / \infty$ & $82 / \infty$ & $324 / \infty$ & $164 / 100$ & $164 / 10$ & $164 / 1$ \\
\hline $3.0-3.1$ & 0.94 & 0.96 & 0.90 & 0.92 & 0.67 & 0.05 \\
$3.6-3.8$ & 0.98 & 0.98 & 0.98 & 0.96 & 0.64 & 0.03 \\
$3.0-4.0$ & 0.91 & 0.92 & 0.89 & 0.88 & 0.61 & 0.03 \\
$4.9-5.1$ & 0.95 & 0.96 & 0.93 & 0.93 & 0.70 & 0.07 \\
$8.2-9.2$ & 0.96 & 0.96 & 0.96 & 0.95 & 0.86 & 0.38 \\
$10.2-11.2$ & 0.99 & 0.99 & 0.99 & 0.99 & 0.93 & 0.56 \\
$10.0-13.0$ & 0.99 & 0.99 & 0.98 & 0.98 & 0.90 & 0.47 \\
$18.0-22.0$ & 0.89 & 0.93 & 0.85 & 0.89 & 0.77 & 0.27 \\
$20.0-20.2$ & 0.98 & 0.99 & 0.97 & 0.98 & 0.85 & 0.30 \\
$24.1-24.9$ & 0.92 & 0.96 & 0.85 & 0.91 & 0.83 & 0.38 \\
$32.0-32.5$ & 0.48 & 0.67 & 0.24 & 0.47 & 0.44 & 0.27 \\
$220-230$ & 0.21 & 0.39 & 0.07 & 0.21 & 0.21 & 0.20 \\
$330-370$ & 0.56 & 0.66 & 0.41 & 0.56 & 0.56 & 0.54 \\
$430-470$ & 0.61 & 0.71 & 0.46 & 0.61 & 0.61 & 0.60 \\
\hline
\end{tabular}

model, as well as for two variations, one with the relative humidity at each layer of the atmosphere in the model doubled, and another with the relative humidity halved. The resulting emission and transmission spectra are shown in Figures 3-6, for the wavelength ranges 2$6 \mu \mathrm{m}, 5-15 \mu \mathrm{m}, 15-60 \mu \mathrm{m}$ and $50-500 \mu \mathrm{m}$, respectively. Tables 2 and 3 give some numerical values for the sky flux and transmission, averaged over several wavelength regions of interest.

MODTRAN calculates the total vapour column of absorption $h$, for a species such as water, as

$$
h=\frac{1}{\rho_{0} R m_{a}} \int U r_{w}(P, T) \frac{P}{T} d z,
$$

where $U$ is the relative humidity, $P$ is the pressure and $T$ the temperature of the air, and $r_{w}$ is the saturation mixing ratio of moist air (i.e. the mass of water in saturated air compared to the equivalent mass of dry air that contains it), all as functions of $z$, the height. Here $\rho_{0}$ is the density of dry air at STP, $m_{a}$ the molecular mass of air and $R$ the gas constant. The column $h$ can also be related to the precipitable water vapour content $X$ by

$$
h=\frac{\rho_{w} m_{a}}{\rho_{0} m_{w}} X
$$

or $h(\mathrm{~cm})=0.12 X(\mu \mathrm{m})$, where $\rho_{w}$ is the density of liquid water at STP and $m_{w}$ its molecular mass $\left(\mathrm{g} \mathrm{mol}^{-1}\right)$. 

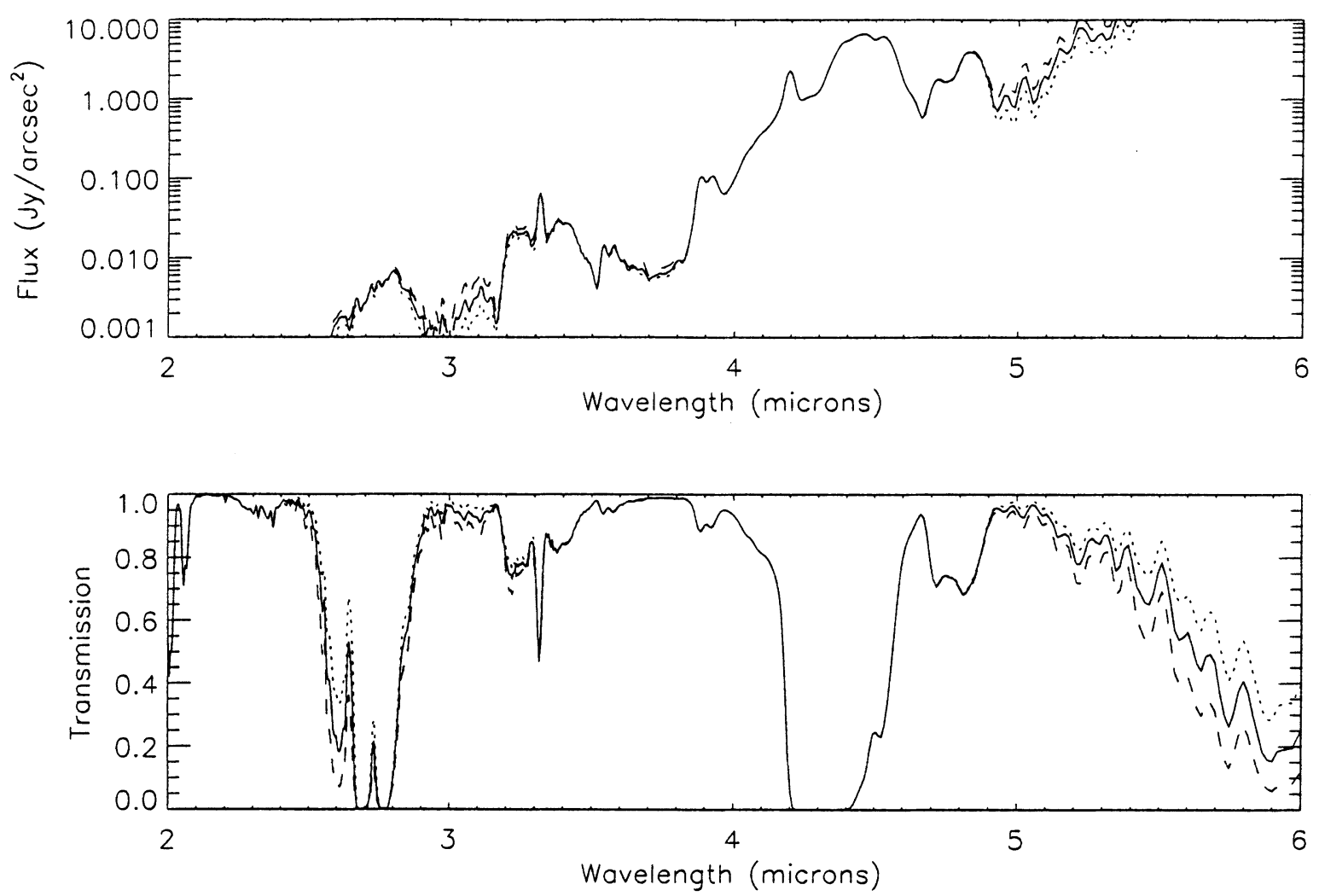

Figure 3-Comparison of emission and transmission spectra with varying water vapour content in the near-IR, from 2 to $6 \mu \mathrm{m}$. The solid curve is the spectrum for the original model (profiles shown in Figure 1), with total precipitable water content $164 \mu \mathrm{m}$. The dashed curves (upper curve on the emission spectrum, lower curve on the transmission spectrum) are for the same model, but with almost double the water content $(324 \mu \mathrm{m})$, while the dotted curves (lower and upper curves, respectively) are for when the water vapour content is halved $(82 \mu \mathrm{m})$.
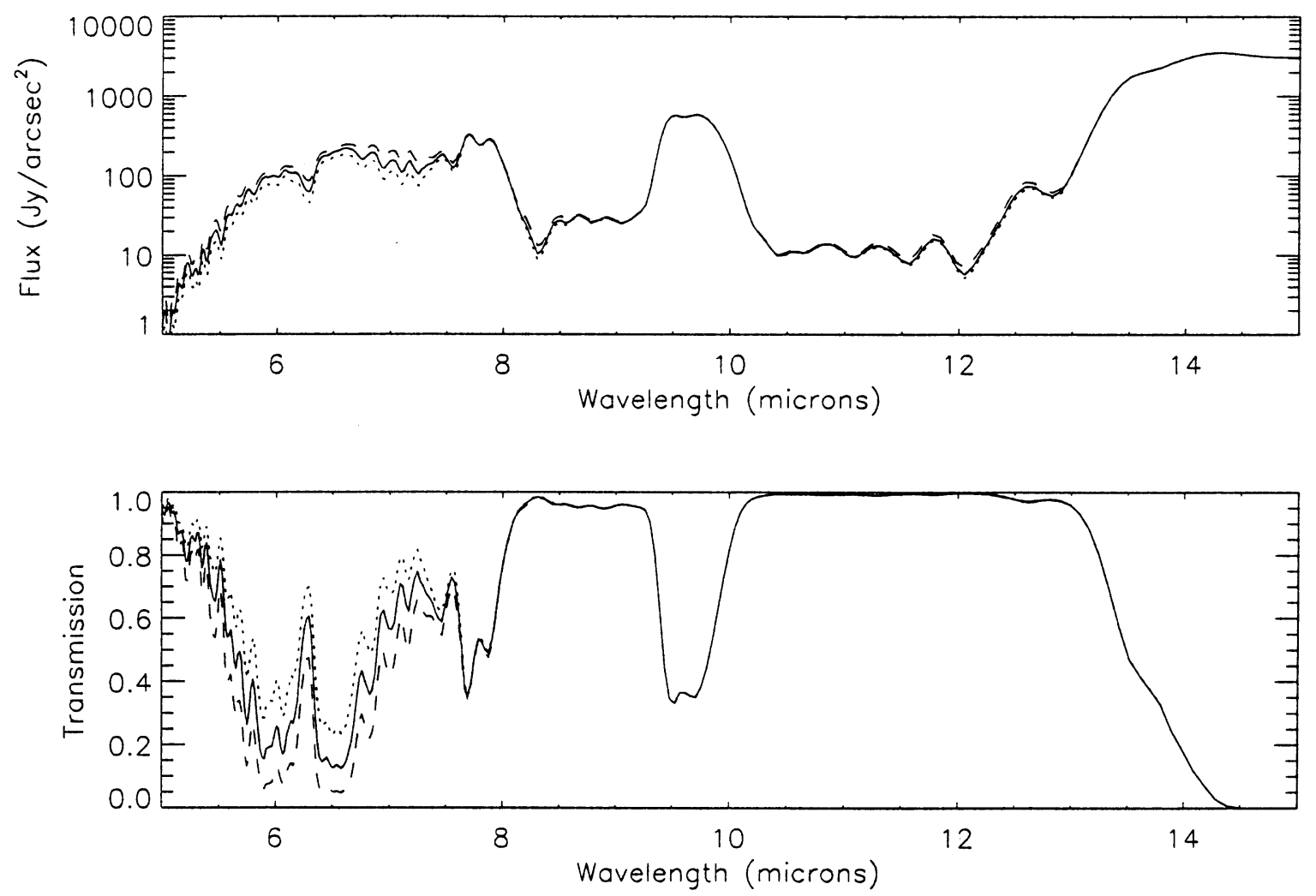

Figure 4-Comparison of emission and transmission spectra with varying water vapour content in the mid-IR, from 5 to $15 \mu \mathrm{m}$, as for Figure 3. Note that the flux range is different. 

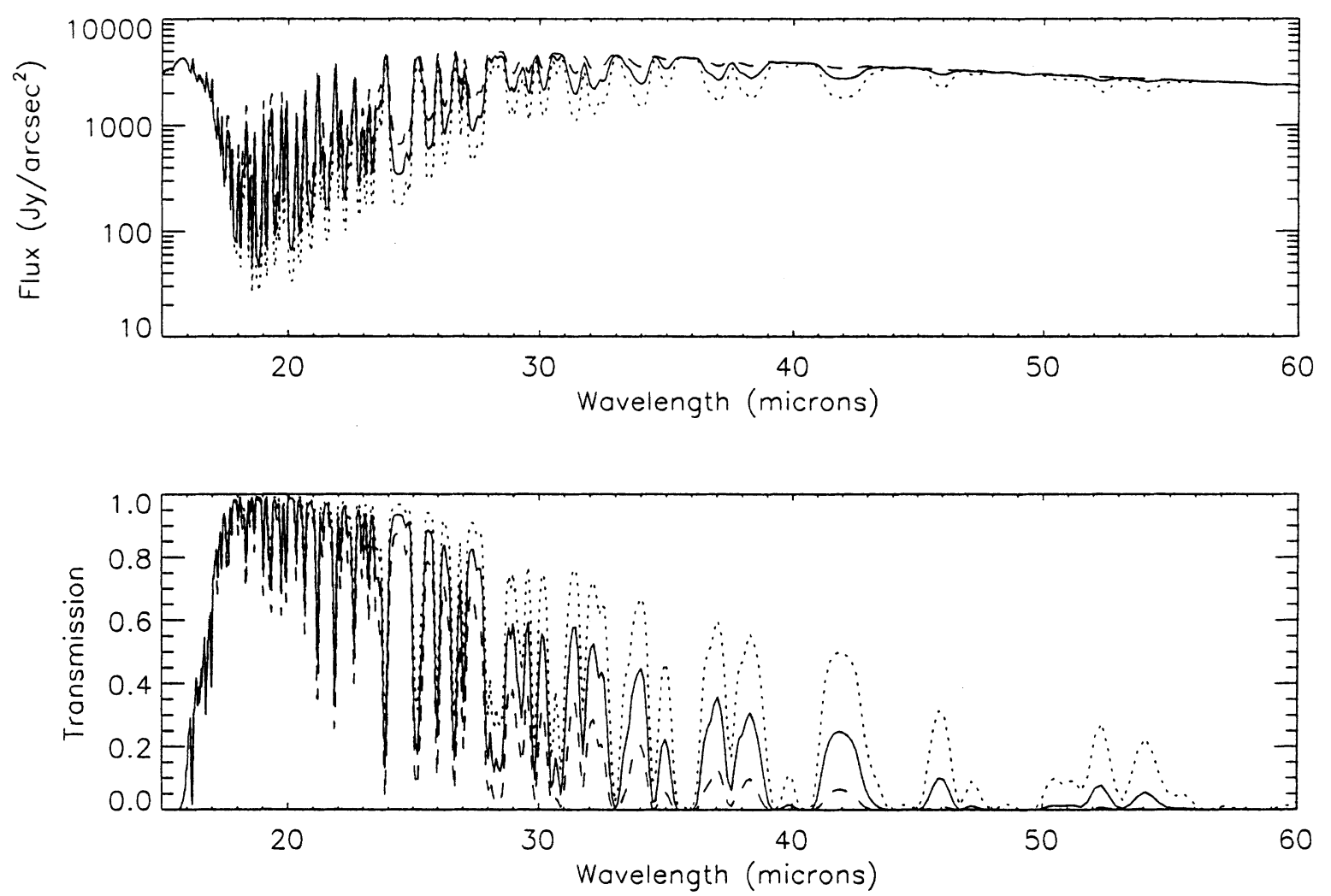

Figure 5-Comparison of emission and transmission spectra with varying water vapour content in the mid-IR, from 15 to $60 \mu \mathrm{m}$, as for Figure 3. Note that the flux range is different.
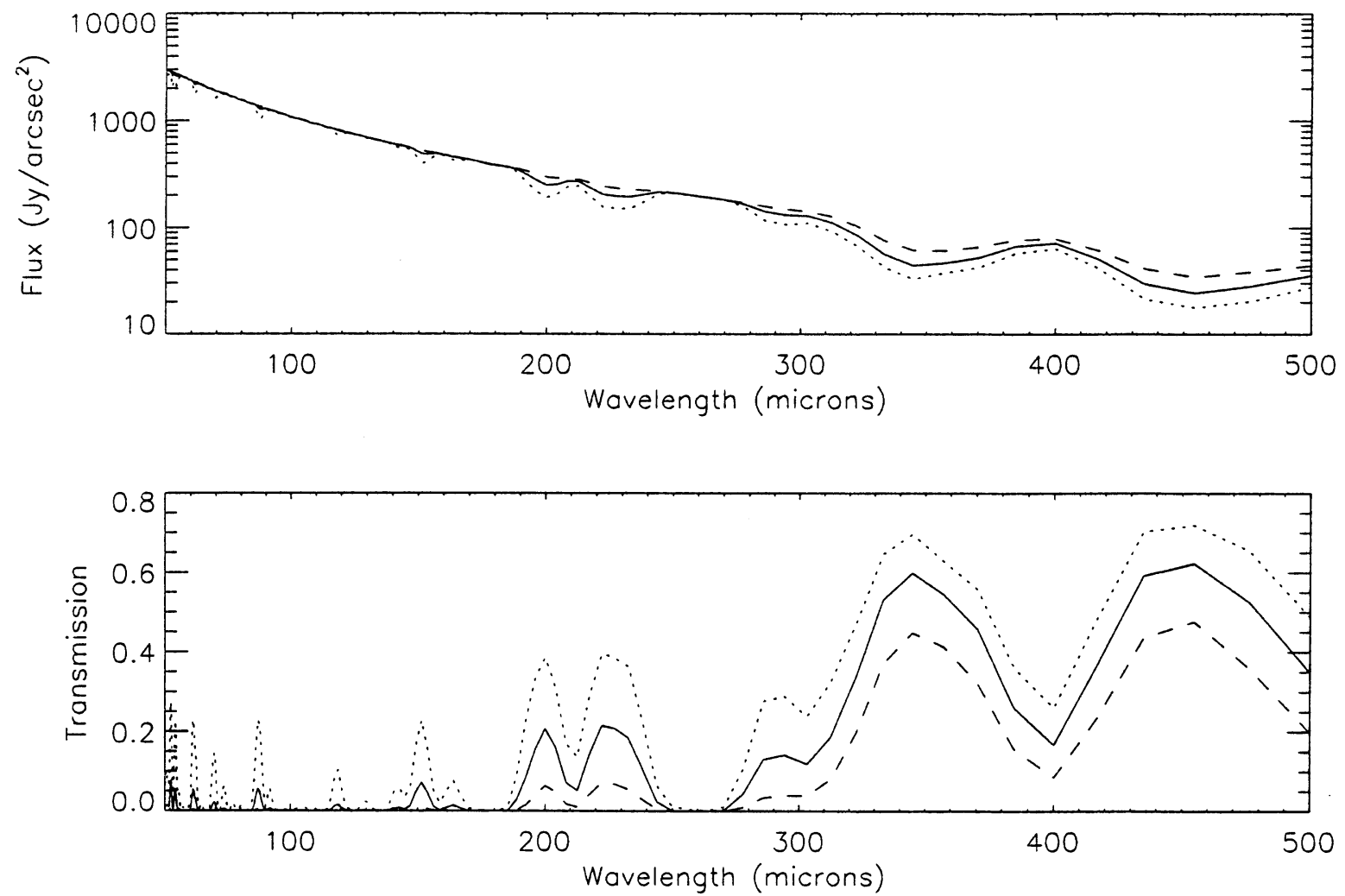

Figure 6-Comparison of emission and transmission spectra with varying water vapour content in the far-IR and sub-mm, from 50 to $500 \mu \mathrm{m}$, as for Figure 3. Note that both the flux and transmission ranges are different. 
Note that part of the reason for the low water vapour content of the atmosphere above Antarctica is that at these low temperatures the saturation vapour pressure of water is low. For instance, while $r_{w}$ is $8 \mathrm{~g} \mathrm{~kg}^{-1}$ at $10^{\circ} \mathrm{C}$ and $1000 \mathrm{mbar}$, it is only $0.2 \mathrm{~g} \mathrm{~kg}^{-1}$ at $-40^{\circ} \mathrm{C}$ and $600 \mathrm{mbar}$. Therefore, even when the relative humidity is close to $100 \%$, the actual amount of water vapour is still small. When the relative humidity profile of the original model was doubled, it actually exceeded $100 \%$ at one level, and had to be reset to $100 \%$. This is why the 'wet' case does not have exactly twice the precipitable water. In fact, even scaling the relative humidity up by an unphysical factor of 10 only resulted in $540 \mu \mathrm{m}$ of precipitable water.

Halving and doubling the humidity in this way encompasses the range in precipitable water vapour content encountered at the South Pole. For instance, Chamberlin et al. (1997) show that in winter there is less than $190 \mu \mathrm{m}$ of precipitable water vapour for $25 \%$ of the time, and less than $320 \mu \mathrm{m}$ for $75 \%$ of the time. Even in summer the column is less than $470 \mu \mathrm{m}$ for half the time. Furthermore, the precise determination of water vapour content from radiosonde data is difficult below $-40^{\circ} \mathrm{C}$ (e.g. see Walden, Warren \& Murcray 1998), and so encompassing the likely range in this way allows more robust conclusions to be drawn than detailed modelling of several sets of radiosonde data.

From $2-15 \mu \mathrm{m}$ there is relatively little variation in either transmission or flux as the water vapour content is varied (except within the water absorption band itself around $6 \mu \mathrm{m})$. It seems, therefore, that at a very dry site such as the Antarctic Plateau, the exact value of the water content is not an important factor in determining the observing conditions in the dark regions of the nearand mid-IR spectrum.

For wavelengths beyond $20 \mu \mathrm{m}$, however, it is a different story. The opening of the mid-IR window at $30 \mu \mathrm{m}$ is critically dependent on low levels of water vapour, being effectively closed for $324 \mu \mathrm{m}$ of precipitable water vapour. While the background fluxes do not vary significantly with water vapour content, the transmission, and thus ability to detect a signal, does. In particular, the new far-IR windows at 200 and $230 \mu \mathrm{m}$ do require the driest days for successful observing. However, the only terrestrial locations from which these windows are accessible at all are probably those on the Antarctic Plateau.

\section{The Effect of Aerosols}

Chamberlain et al. (2000) found that the modelled flux in the dark windows of the mid-IR (8.2-9.2 and 10.2$12.2 \mu \mathrm{m}$ ) was strongly dependent on the amount of aerosols included in the model. One of MODTRAN's standard aerosol types, 'radiative fog', was used in these models. To investigate the effects of aerosols on the transmission and emission, models with different visibilities were calculated. Starting with that used above for $164 \mu \mathrm{m}$ of precipitable water vapour (see Figure 1), increasing amounts of 'radiative fog' were added, cor-
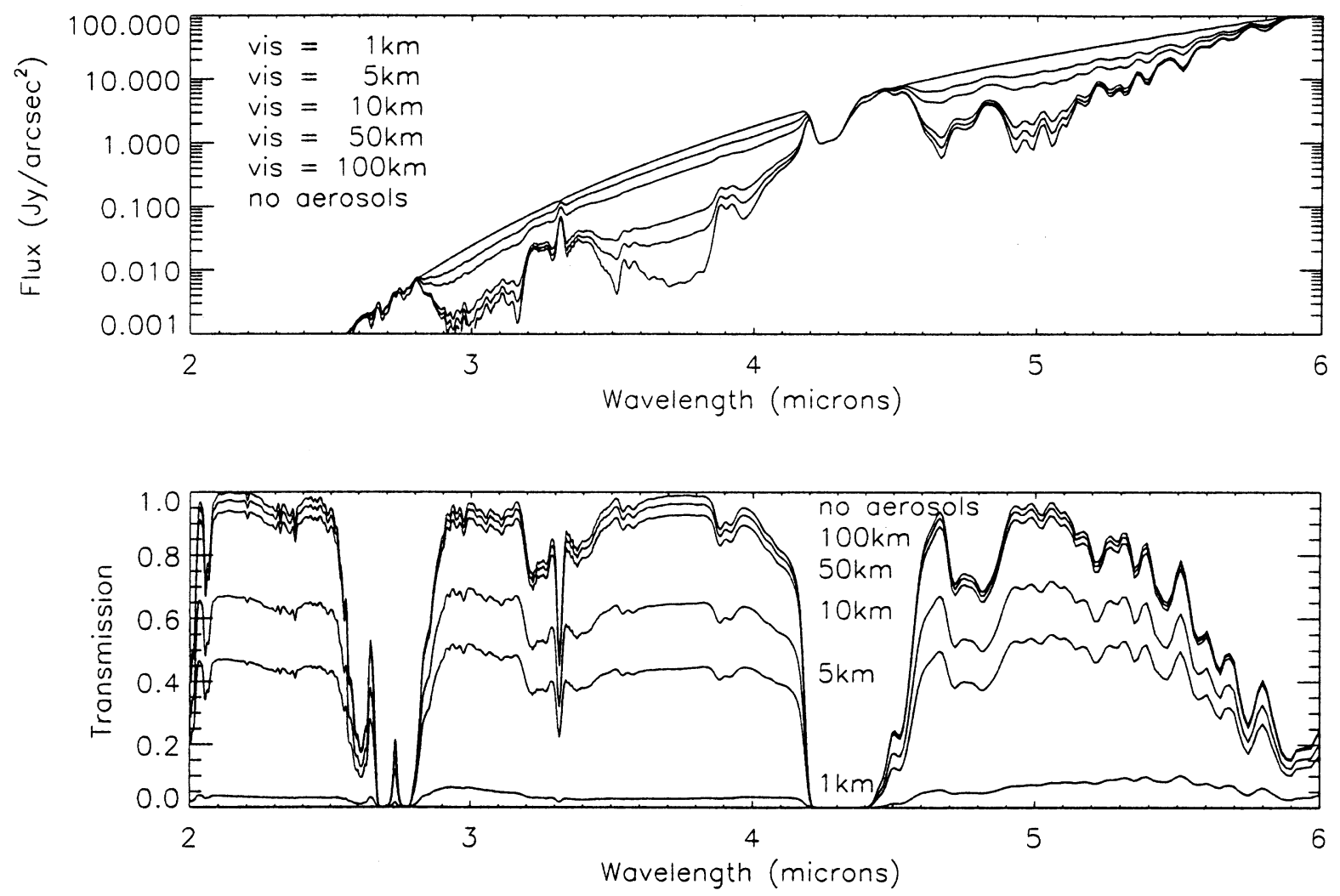

Figure 7-Emission and transmission spectra for models with varying visibility of aerosols, as labelled (no aerosols, 100, 50, 10, 5 and $1 \mathrm{~km})$, in the near-IR from $2-6 \mu \mathrm{m}$. The model has $164 \mu \mathrm{m}$ of precipitable water vapour, as in Figure 1. 

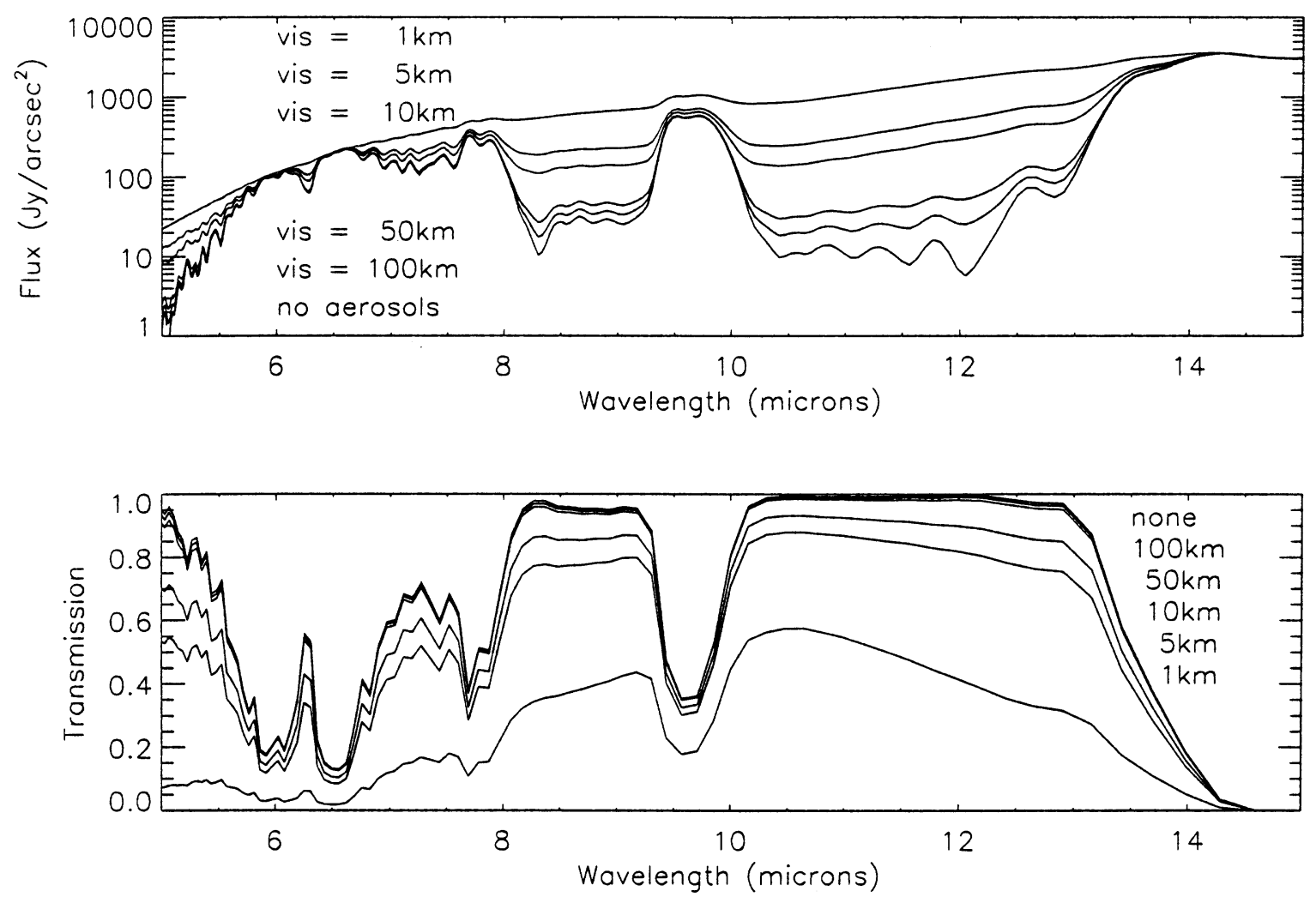

Figure 8-As for Figure 7, emission and transmission spectra for models with varying visibilities, in the mid-IR from 5 to $15 \mu \mathrm{m}$ (note the difference in flux range).
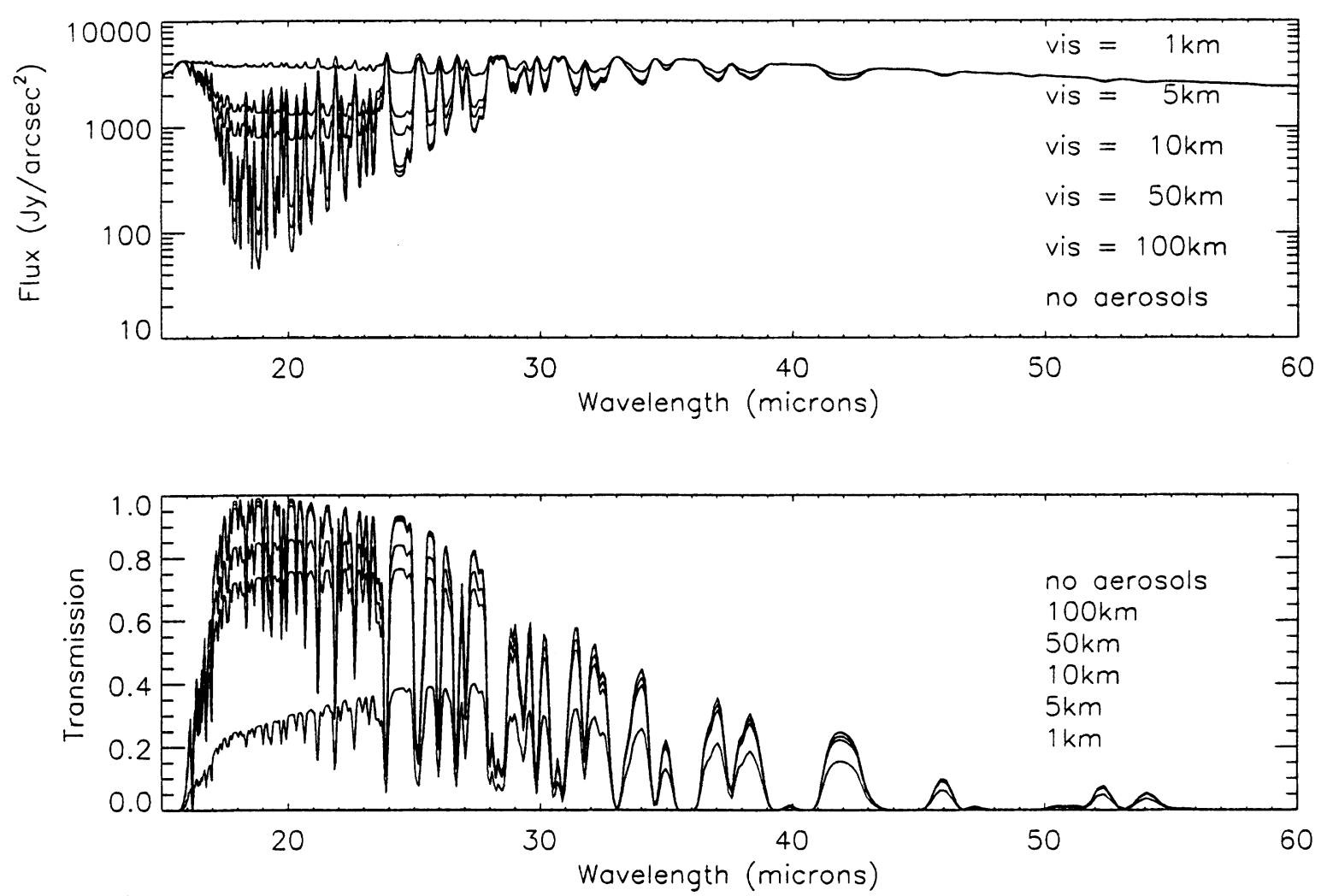

Figure 9-As for Figure 7, emission and transmission spectra for models with varying visibilities, in the mid-IR from 15 to $60 \mu \mathrm{m}$ (note the difference in flux range). 

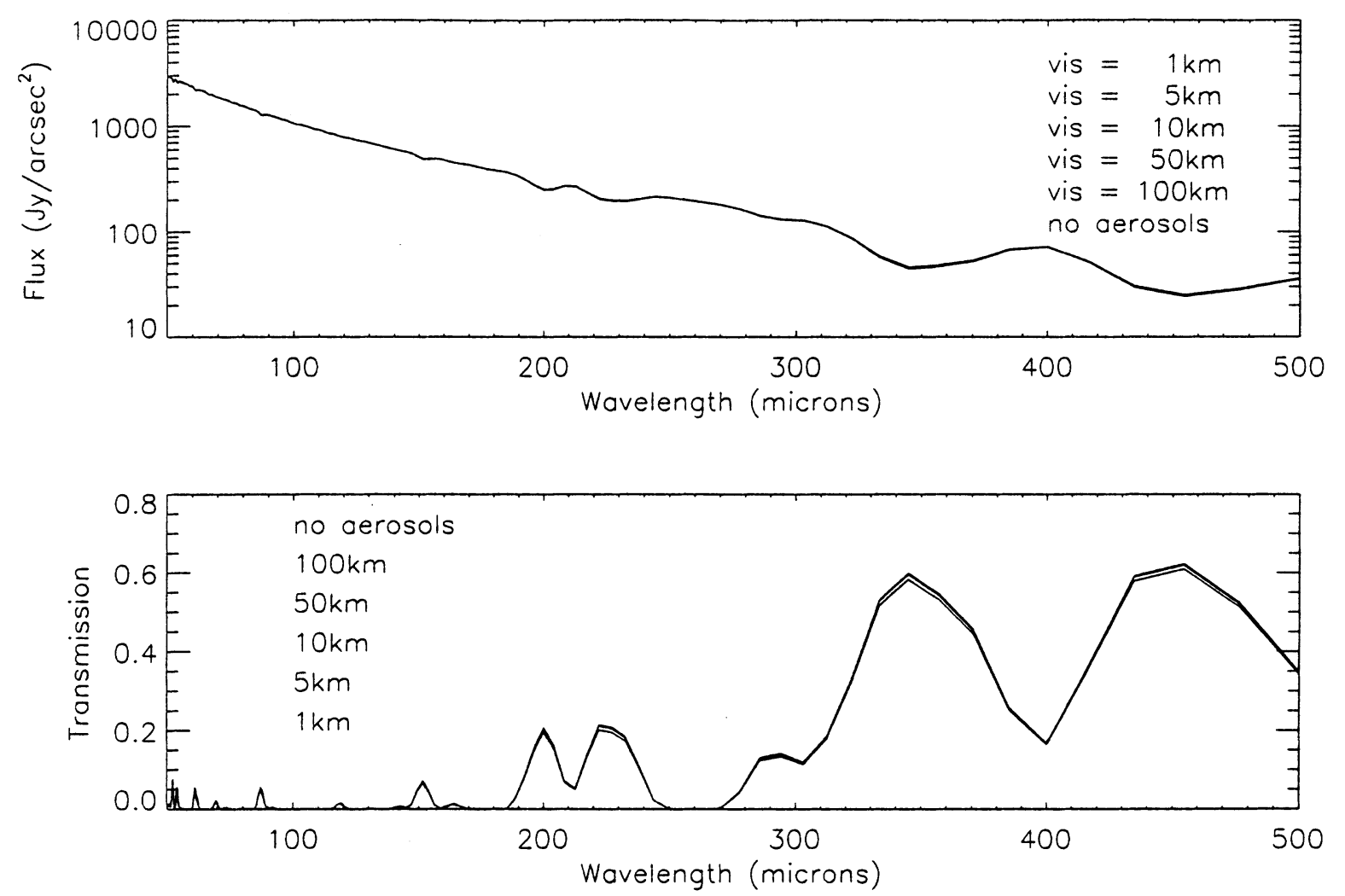

Figure 10-As for Figure 7, emission and transmission spectra for models with varying visibilities, in the far-IR and sub-mm from 50 to $500 \mu \mathrm{m}$ (note the differences in flux range and transmission range).

responding to visibilities of 'clear' (no aerosols), then $100,50,10,5$ and $1 \mathrm{~km}$. Figures 7-10 show the resulting spectra in the same wavelength ranges as before. Tables 2 and 3 give some numerical values for the sky flux and transmission for visibilities of $1,10,100 \mathrm{~km}$ and 'clear', averaged over several wavelength regions of interest.

For a visibility of $1 \mathrm{~km}$, the sky is almost a perfect greybody at all wavelengths, with a temperature of approximately $-43^{\circ} \mathrm{C}$ (which is the temperature at the top of the inversion layer). In some cases where the sky is completely optically thick, the flux is actually less than this level, but this corresponds to path lengths so short that they remain close to ice level, where the temperature is some $30^{\circ} \mathrm{C}$ lower.

For wavelengths generally longer than $50 \mu \mathrm{m}$, aerosols have negligible effect on both the transmission and the emission. Since the sky is always rather optically thick here, the emission spectrum is already close to that of a blackbody at the temperature of the lower atmosphere. Therefore the aerosols, which are additional emitters at the same temperature, cannot increase the flux significantly. This is also the case in the optically thick parts of the mid-IR spectrum (in the water, ozone and carbon dioxide bands, at 7.8, 9.3 and $14.0 \mu \mathrm{m}$ respectively).

However, in windows at wavelengths shorter than $50 \mu \mathrm{m}$, aerosols have a considerable effect on the emission and transmission. In the near-IR, visibilities greater than $10 \mathrm{~km}$ are needed for good observing conditions. The transmission is no better than $60 \%$ for a visibility of $10 \mathrm{~km}$, with the flux levels close to their blackbody value. In the mid-IR windows, while flux levels increase significantly from their lowest values for a visibility of $10 \mathrm{~km}$, the transmission itself is not seriously degraded unless the visibility falls below $5 \mathrm{~km}$. When the visibility is $50 \mathrm{~km}$ or better, the mid-IR windows at $8-9,10-12$ and around $20 \mu \mathrm{m}$ are particularly clear.

The aerosols have been modeled as 'radiative fog'. We note, however, that they do not necessarily correspond to any physical aerosol observed in the Antarctic atmosphere. The aerosols affecting observing conditions in Antarctica are suspected to consist of ice crystals ('diamond dust') within the inversion layer, or possibly within cirrus clouds. However, very little is currently known about the exact properties, origin and location within the atmosphere of these particles.

Nevertheless, it is clear from the above discussion that the effect of aerosols in the sub-mm will be insignificant, regardless of their exact nature. In addition, the ice crystals (if indeed that is what the aerosols are) have physical dimensions of order $10 \mu \mathrm{m}$, so at sub-mm wavelengths they will behave as simple scatterers. Smith \& Harper (1998) reported a small increase in flux levels at $10 \mu \mathrm{m}$ during a short period of visible diamond dust during daytime measurements. Van Allen et al. 
(1996) also note ever-present levels of blowing snow from measurements they made at the South Pole with a Fourier transform spectrometer. If these are the main contributors to the aerosols, then on the summit of the Plateau, where wind levels are lower, the visibility would generally be expected to be higher than at the Pole and therefore the site would be superior for near-IR and mid-IR observations. At the present time, no IR measurements have been made on the higher Plateau, although Valenziano \& Dall'Oglio (1999) report good sub-mm conditions at Dome C.

\section{Conclusions}

Modelling the IR and sub-mm sky fluxes and atmospheric transmission at the South Pole, and examining their sensitivity to temperature, water vapour content and aerosol level, we reach the following conclusions:

(1) At near-IR wavelengths atmospheric temperature has the dominant effect on flux levels in astronomical observing windows. The temperature at the top of the inversion layer, rather than at ice level, determines the amount of the thermal emission. The amount of aerosols, however, has a significant effect on both the atmospheric flux and the transmission. Water vapour content, over the range of values encountered on the Antarctic Plateau, plays little role in determining these levels.

(2) In the mid-IR windows, up to and including the $20 \mu \mathrm{m}$ window, aerosol content plays the primary role in determining flux and transmission levels for the atmosphere. Temperature plays a secondary role in determining the flux levels. Water vapour content has a marginal role.

(3) In the $30 \mu \mathrm{m}$ mid-IR window, the far-IR windows at $200 \mu \mathrm{m}$ and $230 \mu \mathrm{m}$, and in the sub-mm, water vapour content determines the atmospheric transmission. Aerosols play a negligible role. Flux levels only show a small range of variation with water vapour content, instead being set by the temperature. Flux levels are directly proportional to absolute temperature.

We stress that these conclusions are valid only for exceptionally cold, dry sites, such as those on the Antarctic Plateau. The important qualities of an Antarctic observing site are high altitude, and a dry, stable and cold atmosphere, clear of cloud and aerosols. Our results show that these factors affect different wavebands to different extents. Therefore, the choice of optimum site within the continent may depend upon the type of observation being considered. Minimising the aerosol content can clearly be an important factor in this choice.

\section{Future Work}

Several questions regarding the source of emission in the mid-IR remain unanswered. It is clear that aerosols play a vital role. However, the exact nature of these aerosols, including their origin and distribution throughout the atmosphere, is still unknown. A better understanding of these details may help to identify possible sites where the effect of aerosols is minimised, thus improving observing conditions. One possibility is to use a LIDAR to measure the altitude at which aerosols are located. This instrument uses backscattered laser light to probe the atmosphere. It could also help determine the origin of the aerosol particles, and whether they are related in any way to visible cloud.

\section{Acknowledgments}

This research is supported by the Australian Research Council through the Large Grant scheme. We also thank Michael Ashley, Renate Heugberger, Simon Radford and Jeff Peterson for useful discussions, and for allowing us to examine their data before publication.

\section{References}

Ade, P. A. R., Chamberlin, R. A., O’Kelly, M. J., Peterson, J. B., Radford, S. J. E., \& Schartman, E. 2000, Comparison of the Submillimeter Opacity and Sky Stability at Mauna Kea, Chajnantor and South Pole, (in preparation)

AFRL/VSBM, 29 Randolph Road, Hanscom AFB, MA 017313010, USA, http://www-vsbm.plh.af.mil/

Ashley, M. C. B., et al. 1996, PASP, 108, 721

Burton, M. G. 1998, in Astrophysics from Antarctica, ASP Conf. Ser. 141 (San Francisco: ASP), p. 3

Chamberlain, M. A., Ashley, M. C. B., Burton, M. G., Phillips, A., Storey, J. W. V., \& Harper, D. A. 2000, ApJ (in press)

Chamberlin, R. A., Lane, A. P., \& Stark, A. A. 1997, ApJ, 476, 428

CMDL/NOAO (Climate Monitoring \& Diagnostics Laboratory/ National Oceans Atmosphere Organisation), 325 Broadway R/ E/CG, Boulder, CO 80303, USA, http://www.cmdl.noaa.gov/ spo/

Nguyen, H. T., et al. 1996, PASP, 108, 718

Phillips, A., et al. 1999, ApJ, 527, 1009

Smith, C. H., \& Harper, D. A. 1998, PASP, 110, 747

Valenziano, L., \& Dall'Oglio, G. 1999, PASA, 16, 167

Van Allen, R., Murcray, F. J., \& Liu, X. 1996, Appl. Optics, 35, 1523

Walden, V. P., Warren, S. G., \& Murcray, F. J. 1998, J. Geophys. Res., 103, 3825 